\section{COGNITIVE OUTCOME AT SCHOOL-AGE IN CHILDREN WITH POST-HAEMORRHAGIC HYDROCEPHALUS}

J.C. Holwerda' ${ }^{1}$, K.N. Van Braeckel ${ }^{1}$, E. Roze ${ }^{1}$, E.W. Hoving ${ }^{2}$, C.G. Maathuis ${ }^{3}$, O.F. Brouwer ${ }^{4}$,

$$
\text { A.F. Bos }{ }^{1}
$$

${ }^{1}$ Neonatology, Beatrix Children's Hospital, University Medical Center Groningen,

${ }^{2}$ Neurosurgery, ${ }^{3}$ Physiatry, ${ }^{4}$ Child Neurology, University Medical Center Groningen, Groningen, The Netherlands

Objective: Preterm infants are at risk for intracranial haemorrhage with subsequent hydrocephalus. Post-haemorrhagic hydrocephalus is associated with poor outcome but little is know about the long-term outcome at school-age. Our aim was to determine the cognitive outcome in children with post-haemorrhagic hydrocephalus at school-age.

Methods: We included infants treated at the NICU in Groningen with post-haemorrhagic hydrocephalus, born between 1996 and 2003. We reviewed the medical charts for neonatal characteristics and interventional surgery. We determined intelligence (WISC-III-NL), attention (Test of Everyday Attention in Children), and verbal memory (Auditory Verbal Learning Test).

Results: We included 28 children (18 boys, 10 girls), median gestational age 29 weeks (range 25-40) and birth weight 1355 grams (880-3810). Seven children died in infancy. Eleven children of 21 survivors required surgical intervention. At follow-up (median age 11y, [6-14]), 2 children had severe cerebral palsy and were not able to perform cognitive testing. Median total IQ was 88 (59-110) with verbal IQ 88 (62-128) and performance IQ 88 (52-112). Attention scores were normal in 6 (>P15), subclinical (P5-P15) in 1, and abnormal (<P5) in 7 children. Verbal memory was normal in 13, subclinical in 2, and abnormal in 2 children. There was no difference in cognitive scores between children requiring intervention and not.

Conclusion: Children with post-haemorrhagic hydrocephalus have approximately 0.8 SD lower scores on IQ than the norm population. Attention was affected in more than half of the children, verbal memory seemed less affected. Surgical intervention for hydrocephalus was not associated with cognitive outcome.

\section{A CLINICAL PREDICTION MODEL FOR HISTOLOGICAL CHORIOAMNIONITIS AT BIRTH}

\author{
J.V. Been ${ }^{1}$, B.W. Kramer ${ }^{1}$, I.G. Rours ${ }^{2}$, \\ R.F. Kornelisse ${ }^{2}$, R.R. de Krijger ${ }^{3}$, \\ L.J. Zimmermann ${ }^{1}$
}

${ }^{1}$ Paediatrics, Maastricht University Medical Centre, Maastricht, ${ }^{2}$ Paediatrics, ${ }^{3}$ Pathology, Erasmus MC, Rotterdam, The Netherlands

Background: Antenatal exposure to histological chorioamnionitis affects neonatal outcome. Early identification of affected infants could potentiate early intervention. However, in clinical practice the final results of placental pathology may take weeks.

Methods: Placental pathology and relevant clinical data were obtained from consecutively born singleton infants (gestational age $<32$ wks) in the Erasmus MC. Prediction models for histological chorioamnionitis $(\mathrm{HC})$ and for $\mathrm{HC}$ with fetal involvement (FI) were constructed using clinical variables known at birth, in a backward logistic regression model. ROC curves were computed using model-derived predictives.

Results: Of the 216 included infants 84 had $\mathrm{HC}$, of whom 51 had $\mathrm{FI}$. HC+FI was best predicted by a combination of low gestational age, clinical chorioamnionitis, PPROM, absence of preeclampsia and not being small for gestational age (Table 1; $\mathrm{AUC}(95 \% \mathrm{Cl})=0.93(0.89-0.96)$, $\mathrm{p}<.001)$. HC was best predicted by the same model with addition of placental weight (Table 2; AUC $(95 \% \mathrm{Cl})=0.95(0.93-0.98) ; p<.001)$. At a set specificity of $90 \%$, sensitivity of the model is $82 \%$ for $\mathrm{HC}+\mathrm{Fl}$ and $85 \%$ for $\mathrm{HC}$.
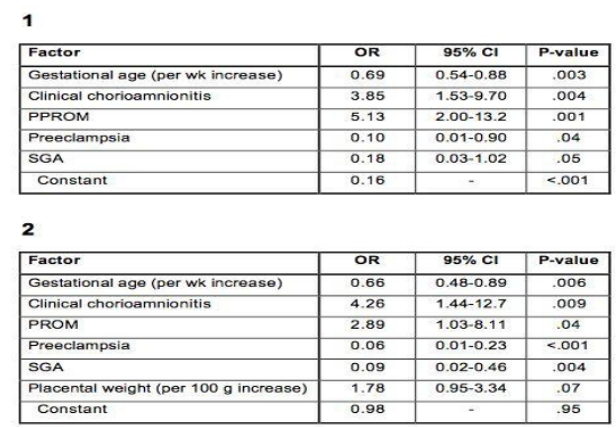

Tables 1+2. Clinical prediction model for histological chorioamnionitis with fetal involvement (1) or any histological chorioamnionitis (2) at birth.

[Tables] 
Poster Presentation Abstracts

Conclusion: $\mathrm{HC}$ and $\mathrm{HC}+\mathrm{FI}$ can be predicted fairly accurately by a simple set of clinical variables available at birth. External validation is required to confirm the usefulness of these models. This may potentiate early intervention to improve future outcome in affected infants.

\section{2}

\section{VALIDATION OF AN OBSERVATIONAL RATING SCALE OF PARENTAL INTERACTIONS WITH VERY PRETERM INFANTS}

\author{
A. Mitchell ${ }^{1}$, C. Beer ${ }^{1}$, S. Johnson ${ }^{1,2}$, \\ C. Glazebrook ${ }^{1}$
}

${ }^{1}$ The University of Nottingham, Nottingham, ${ }^{2}$ UCL Institute for Women's Health, London, UK

Objective: To date studies exploring the influence of maternal interactions on promoting cognitive and emotional development have relied on study specific scales or used the highly regarded Nursing Child Assessment Teaching Scale (NCATS). This study will validate a new, brief and easy to use measure developed to assess the quality of early maternal interactions.

Method: Participants were 43 mothers and their very preterm infants ( $<32$ weeks gestation) selected from a representative sample recruited in a previous study. Mothers were videoed undertaking a teaching task with their infant at 3-months corrected age and mother-infant interaction was assessed using the NCATS (73 items). In the present study interactions were reassessed using the purpose-developed 8 item Observational Scale for Parent Interaction (OSPI), possible scores range from 0 to 15 .

Results: OSPI scores ranged from 1 to 15 , with a median score of 7 . The scale had good internal consistency Cronbach's alpha $=0.758$. OSPI scores correlated significantly with the NCATS Caregiver score $(r=0.781, n=43, p<0.001)$, and with the NCATS Caregiver and Child total score $(r=0.772$, $n=43, p<0.001$ ) demonstrating concurrent validity. Infant's gestational age correlated significantly with the OSPI $(r=0.448, n=43, p=0.003)$ but not with mother's age indicating construct validity. Ten interactions were re-rated using the OSPI and the intraclass correlation of 0.904 shows excellent test re-test reliability.

Conclusions: The OSPI is a valid and reliable measure of maternal interactions which is cost and time efficient. The scale should prove a useful measure particularly for larger studies.
453

\section{LIMITED SIGNIFICANCE OF WEIGHT GAIN DURING PREGNANCY ON BIRTH WEIGHT}

J.H. Grundt ${ }^{1}$, J.O. Nakling ${ }^{2}$, T. Markestad ${ }^{3}$

${ }^{1}$ Pediatrics, ${ }^{2}$ Gynecology \& Obstetrics, ${ }^{3}$ Research Unit, Innlandet Hospital Trust, Lillehammer, Norway

Background and aim: Data from The Medical Birth Registry of Norway show a temporary increase in mean birth weight and proportion of large babies in parallel with changes in national consumption of sugar-containing beverages. The aim was to study to what extent weight increase during pregnancy, which may reflect consumption of carbohydrates, affects birth weight.

Methods: During 2001-2009 15054 live term (3742 weeks) singleton pregnancies were registered in the Oppland county pregnancy database. Time trends were examined, and 9282 births with complete information were subjected to multiple linear regression analysis. Birth weight (BW) was related to age, height, pre-pregnancy BMI (ppBMI), chronic diseases, parity, pregnancy complications, smoking and gestational weight gain (GWG) in the mother, and gestational age (GA), sex, and registered malformations in the child.

Results: During the study period mean BW decreased from 3658 to 3574 gram $(p<001)$. Mean ppBMl increased from 24.25 to 24.78 , GWG decreased from 15.15 to $13.93 \mathrm{~kg}$, smokers decreased from 22.7 to $11.5 \%$, GA decreased from 39.79 to 39.50 weeks, $(p<001$ for all). Our model explained $33 \%$ of the variability in BW. BW increased $18.9(95 \% \mathrm{Cl} ; 17.4,20.4) \mathrm{g}$ per kg GWG, $21.7(19.9,23.5) \mathrm{g}$ per unit ppBMI, $13.1(11.7,14.4) \mathrm{g}$ per $1 \mathrm{~cm}$ mother's height, 141.8 (135.0-148.6) g per week GA, and $105.7(89.3-122.2) \mathrm{g}$ for male sex, and decreased $187.3(164.6,210.1) \mathrm{g}$ for smoking and $182.7(164.5,200.9) \mathrm{g}$ for being primipara.

Conclusion: Pregnancy weight gain and prepregnancy BMI have significant, but limited, impact on birth weight. 